Article

\title{
Galaxy Formation and Rotation as a 4D Relativistic Cloud-World Embedded in a 4D Conformal Background: A Cloud Theory
}

\author{
Mohammed B. Al-Fadhli 1,* \\ 1 College of Science, University of Lincoln, Lincoln, LN6 7TS, UK. \\ * Correspondence: malfadhli@lincoln.ac.uk; mo.fadhli7@gmail.com.
}

\begin{abstract}
The recent observation of the G2 gas cloud orbit around the galactic centre has challenged the model of a mere supermassive black hole at the centre of our galaxy which should have destroyed it. In addition, the Planck Legacy 2018 (PL18) release has preferred a positively curved early Universe with a confidence level exceeding $99 \%$. In this study, the collapse of a large gas cloud in the early Universe to form a galaxy is modelled based on extended field equations as a $4 \mathrm{D}$ relativistic CloudWorld that flows and spins through a 4D independent conformal background of an initial positive curvature considering the preference of the PL18 release. Owning to the curved background, this scenario of galaxy formation indicates that the core of the galaxy undergoes a forced vortex formation with a central event horizon leading to opposite traversable wormholes that are spatially shrinking through the conformal time. It reveals that the galaxy and its core are formed at the same process where the surrounding gas clouds form the spiral arms due to the frame-dragging induced by the fast-rotating core. Accordingly, the G2 gas cloud that only faced the drag effects could be explained if its orbit is around the wormhole but at a distance from the central event horizon. The formation of the galaxy and its core simultaneously could explain the formation of the supermassive compact galaxy cores with a mass of $\sim 10^{9} \mathrm{M} \odot$ at just $6 \%$ of the current Universe age and could resolve the black hole hierarchy problem.
\end{abstract}

Keywords: Galaxy Formation; Conformal Space-time Background.

\section{Introduction}

Observations from the Deep Extragalagtic Evolutionary Probe 2 Survey of a large sample of disk galaxies found that the motion of galaxies was steadily getting in order with their rotation velocity increasing over the last eight billion years $[1,2]$. In addition, the Planck Legacy 2018 (PL18) release has confirmed the presence of an enhanced lensing amplitude in the cosmic microwave background power spectra, which prefers a positively curved early Universe with a confidence level greater than $99 \%$ [3,4]. Further, galactic rotation curves were found to be influenced by external fields [5] and follow the baryonic Tully-Fisher relation [6,7]. Furthermore, the Dark Energy Survey Collaboration has recently released the largest maps of galaxy distribution and shapes, which showed that the dark matter is more distributed than that predicted by General Relativity $[8,9]$. On the other hand, several studies reported that some galaxies are missing dark matter [10-12].

To get insights from these observations while aiming to explain the G2 cloud's observation that has challenged the model of a mere supermassive black hole at the centre of our galaxy $[13,14]$, this study presents a new galaxy formation scenario considering the background curvature as preferred by the PL18 release. New field equations are derived that in which the Bulk is regarded as the independent background represented by the $4 \mathrm{D}$ conformal space-time with a distinct curvature evolving over cosmic time; throughout this Bulk, Branes/Cloud-Worlds as 4 D relativistic celestial objects can flow and spin. 


\section{Interactions between the Cloud-World and the Background}

The celestial objects are regarded as 4D relativistic Cloud-Worlds/Branes of local three spatial dimensions and local temporal dimension, where these Cloud-Worlds travel and spin through the Bulk as the independent background that is regarded as the global conformal space-time with distinct curvature that evolves over cosmic time. An action governing the interaction of the stress-energy-momentum density between the CloudWorld and the conformal background is formulated as an extension to the Einstein-Hilbert action as follows

$$
S=\int d^{4} \sigma \sqrt{-g}\left[\frac{T}{\mathcal{T}}+\mathcal{L}\right]
$$

where $T$ is the trace of the stress-energy-momentum tensor $T_{\mu \nu}$ of the $4 \mathrm{D}$ Cloud-World that is embedded in the $4 \mathrm{D}$ conformal space-time as the independent background, represented by the stress-energy-momentum tensor $\mathcal{T}_{\mu \nu}$ of trace $\mathcal{T} . \mathcal{L}$ is the Lagrangian density of the Cloud-World and $g$ is the determinant of its metric tensor $g_{u v}$. This action features a $4 \mathrm{D}$ spin and flow of the Cloud-World through the independent background that in turn can evolve over cosmic time. The term $T / \mathcal{T}$ is considered in terms of energy density. The variation in the action yields

$$
\delta S=\int d^{4} \sigma \sqrt{-g}\left[\frac{\delta T}{\mathcal{T}}-\frac{\delta \mathcal{T}}{\mathcal{T}^{2}} T+\frac{T \delta \sqrt{-g}}{\mathcal{T} \sqrt{-g}}+\delta \mathcal{L}+\mathcal{L} \frac{\delta \sqrt{-g}}{\sqrt{-g}}\right]
$$

The variation in the trace is $\delta T=T_{\mu \nu} \delta g^{\mu v}+g^{u v} \delta T_{\mu \nu}$ while according to Jacobi's formula for the determinant differentiation, the differentiation gives, $\delta \sqrt{-g}=-\sqrt{-g} g_{\mu \nu} \delta g^{\mu \nu} / 2$ [15]; accordingly:

$$
\delta S=\int d^{4} \sigma \sqrt{-g}\left[\begin{array}{c}
\frac{T_{\mu \nu} \delta g^{\mu \nu}+g^{\mu \nu} \delta T_{\mu v}}{\mathcal{T}}-\frac{\mathcal{T}_{\mu \nu} \delta g^{\mu \nu}+g^{\mu \nu} \delta \mathcal{T}_{\mu v}}{\mathcal{T}^{2}} T \\
-\frac{T}{\mathcal{T}} \frac{g_{\mu v}}{2} \delta g^{\mu \nu}+\delta \mathcal{L}-\mathcal{L} \frac{g_{\mu v}}{2} \delta g^{\mu \nu}
\end{array}\right]
$$

According to Einstein field equations, the variation in the stress-energy tensor, $\delta T_{\mu \nu}$, is proportional to the variation in the Ricci curvature tensor, $\delta R_{\mu \nu}$; while according to Palatini identity, $\delta R_{\mu \nu}=\nabla_{\rho}\left(\delta \Gamma_{v \mu}^{\rho}\right)-\nabla_{v}\left(\delta \Gamma_{\rho \mu}^{\rho}\right)$. Thus, $\delta T_{\mu \nu}=k\left(\nabla_{\rho}\left(\delta \Gamma_{v \mu}^{\rho}\right)-\nabla_{v}\left(\delta \Gamma_{\rho \mu}^{\rho}\right)\right)$ where $k$ is a constant. By using the metric compatibility of the covariant derivative, $\nabla_{\rho} g^{\mu \nu}=0$; thus, $g^{\mu v} \delta T_{\mu \nu}=k\left(\nabla_{\rho}\left(g^{\mu v} \delta \Gamma_{\nu \mu}^{\rho}\right)-\nabla_{v}\left(g^{\mu \nu} \delta \Gamma_{\rho \mu}^{\rho}\right)\right)$. Then, the Cloud-World's boundary term as a total derivative can be transformed according to Stokes' theorem with renaming the dummy indices as follows

$$
k \oint_{\mathcal{M}} d^{4} \sigma \sqrt{-g} \frac{\nabla_{\rho}\left(g^{\mu v} \delta \Gamma_{\nu \mu}^{\rho}-g^{\mu \rho} \delta \Gamma_{\sigma \mu}^{\sigma}\right)}{\mathcal{T}}=k \epsilon \oint_{\partial \mathcal{M}} d^{3} \sigma \sqrt{|q|} \frac{K}{\mathcal{T}}=\epsilon \oint_{\partial \mathcal{M}} d^{3} \sigma \sqrt{|q|} \frac{H}{\mathcal{T}}
$$

where $q$ is the determinant of the induced metric tensor on the boundary of the CloudWorld, $H$ is the extrinsic trace of the stress-energy tensor on the boundary, $K$ is the trace of the extrinsic curvature tensor on the boundary, $\epsilon$ equals 1 when the normal $\hat{n}_{u}$ is a spacelike entity and equals -1 when it is a timelike entity. The non-boundary term $k / \mathcal{T}$ acts only as a scalar to the integral transformation. The same approach is applied for the Bulk boundary term. 
Accordingly, the action in Equation (3) is expressed as

$$
\begin{aligned}
\delta S= & \int d^{4} \sigma \sqrt{-g}\left[\frac{T_{\mu \nu} \delta g^{\mu \nu}}{\mathcal{T}}-\frac{T \mathcal{T}_{\mu \nu} \delta g^{\mu \nu}}{\mathcal{T}^{2}}-\frac{T g_{\mu \nu} \delta g^{\mu \nu}}{2 \mathcal{T}}+\frac{2 \delta \mathcal{L}-\mathcal{L} g_{\mu \nu} \delta g^{\mu \nu}}{2}\right]+ \\
& \int d^{3} \sigma \sqrt{|q|}\left[\frac{\epsilon}{\mathcal{T}} H\right]-d^{3} \sigma \sqrt{|p|}\left[\frac{T \epsilon}{\mathcal{T}^{2}} \mathcal{H}\right]
\end{aligned}
$$

where $\mathcal{H}$ is the extrinsic trace of the stress-energy tensor on the conformal boundary term and $p$ is the determinant of the induced metric tensor on the boundary of the Bulk. It is worth noting that some terms are satisfying the criteria that the variation in the action $\delta S$ is with respect to the variation in the inverse metric $\delta g^{\mu \nu}$ excluding the boundary action that still lacks this feature. To achieve the consistency of the action, the variation in the boundary action has to be determined. The variation in the Cloud-World boundary term:

$$
\int d^{3} \sigma \sqrt{|q|}\left(H_{\mu \nu} \delta q^{\mu \nu}-\frac{1}{2} H\left(q_{\mu \nu} \delta q^{\mu \nu}+2 q_{\mu \nu} \frac{\delta H_{\mu \nu}}{\delta q_{\mu \nu} H} \delta q^{\mu \nu}\right)\right)
$$

here $\delta H_{\mu \nu} / \delta q_{\mu \nu} H=\left(\delta H_{\mu \nu} / H_{\mu \nu}\right)\left(q_{\mu \nu} / \delta q_{\mu \nu}\right)=\delta \ln H_{\mu \nu} / \delta \ln q_{\mu \nu}$ resembles the Ricci flow in a normalised form reflecting the conformal evolution in boundary, which can be expressed as a positive function $\Omega^{2}$ based on Weyl's conformal transformation as $\tilde{q}_{\mu \nu}=q_{\mu \nu} \Omega^{2}$ [16]. The non-boundary term $\epsilon / \mathcal{T}$ is left outside, where it can be considered as a scalar. Nevertheless, its high-order variational terms can be incorporated into the conformal transformation function $\Omega^{2}$. The same approach is applied for the Bulk boundary term. Accordingly, the variation in the action is expressed as

$$
\delta S=\begin{aligned}
& \int d^{4} \sigma \sqrt{-g} \delta g^{\mu \nu}\left[\frac{T_{\mu \nu}}{\mathcal{T}}-\frac{T}{\mathcal{T}^{2}} \mathcal{T}_{\mu \nu}-\frac{T}{\mathcal{T}} \frac{g_{\mu \nu}}{2}-\mathcal{L} \frac{g_{\mu \nu}}{2}+\frac{\delta \mathcal{L}}{\delta g^{\mu \nu}}\right]+ \\
& \int d^{3} \sigma \sqrt{|q|} \delta q_{\mu \nu}\left[\frac{\epsilon}{\mathcal{T}}\left(H_{\mu \nu}-\frac{1}{2} H \hat{q}_{\mu \nu}\right)\right]-d^{3} \sigma \sqrt{|p|} \delta p_{\mu \nu}\left[\frac{T \epsilon}{\mathcal{T}^{2}}\left(\mathcal{H}_{\mu \nu}-\frac{1}{2} \mathcal{H} \hat{p}_{\mu \nu}\right)\right]
\end{aligned}
$$

where $\hat{q}_{\mu \nu}=q_{\mu \nu}+2 \tilde{q}_{\mu \nu}$ is the conformal transformation of the induced metric tensor where Einstein spaces are a subclass of the conformal space [17]. By implementing $\epsilon$ as a timelike entity and applying the principle of stationary action yield

$$
\frac{T_{\mu \nu}}{\mathcal{T}}-\frac{T}{\mathcal{T}^{2}} \mathcal{T}_{\mu \nu}-\frac{1}{2} \frac{T}{\mathcal{T}} g_{\mu \nu}+\frac{T\left(\mathcal{H}{ }_{\mu \nu}-\frac{1}{2} \mathcal{H} \hat{p}_{\mu \nu}\right)-\mathcal{T}\left(H_{\mu \nu}-\frac{1}{2} H \hat{q}_{\mu \nu}\right)}{\mathcal{T}^{2}}=E_{\mu \nu}
$$

where $E_{\mu \nu}=T_{\mu \nu} / 2 ; E_{\mu \nu}$ is the stress-energy tensor of the Cloud-World and $T_{\mu \nu}$ is the stress-energy tensor which is by definition proportional to the Lagrangian term as $T_{\mu \nu}$ := $\mathcal{L} g_{\mu \nu}-2 \delta \mathcal{L} / \delta g^{\mu \nu}[15,18]$. The field equations can be simplified to

$$
\frac{T_{\mu \nu}}{\mathcal{T}}-\frac{1}{2} \frac{T}{\mathcal{T}} \hat{g}_{\mu \nu}+\frac{T\left(\mathcal{H}_{\mu \nu}-\frac{1}{2} \mathcal{H} \hat{p}_{\mu \nu}\right)-\mathcal{T}\left(H_{\mu \nu}-\frac{1}{2} H \hat{q}_{\mu \nu}\right)}{\mathcal{T}^{2}}=E_{\mu \nu}
$$

where $\hat{q}_{\mu \nu}=q_{\mu \nu}+2 \tilde{q}_{\mu \nu}$ regarding the conformal transformation of the metric tensor since $\mathcal{T}_{\mu \nu} / \mathcal{T}=\tilde{q}_{\mu \nu}$. The field equations can be utilized to construct the Brane notion to higher dimensions in the form of a local $4 \mathrm{D}$ relativistic Cloud-World of the celestial object of a local intrinsic stress-energy density represented by $T_{\mu \nu}$ and local extrinsic stress-energy on its boundary represented by $H_{\mu \nu}$, which travels through the independent background as the $4 \mathrm{D}$ conformal space-time of a global intrinsic stress-energy density $\mathcal{T}_{\mu \nu}$ and global extrinsic stress-energy density $\mathcal{H}_{\mu \nu}$ on its boundary. The interpretation is visualized in the next Section. 


\section{Galaxy Formation and Rotation}

The large gas cloud that was collapsing and travelling through the curved background as preferred by the PL18 gains high spinning speed induced by the energy flux divergence. The high-speed spinning can transform its hyperspherical core (Figure 1a) into a forced vortex that can be considered as a tower of almost hypercylinders (Figure $2 b)$. Thus, the gravitational contributions come mainly from the extrinsic curvature terms (boundary terms), while the intrinsic curvature terms vanish as hypercylinders have no intrinsic curvature [19]. The corresponding stress-energy terms are rearranged as

$$
\frac{T_{\mu \nu}}{\mathcal{T}}-\frac{1}{2} \frac{T}{\mathcal{T}} \hat{g}_{\mu \nu}=E_{\mu \nu}-\frac{T\left(\mathcal{H}_{\mu \nu}-\frac{1}{2} \mathcal{H} \hat{p}_{\mu \nu}\right)-\mathcal{T}\left(H_{\mu \nu}-\frac{1}{2} H \hat{q}_{\mu \nu}\right)}{\mathcal{T}^{2}} \cong 0
$$

The $4 \mathrm{D}$ Cloud-World (the gas cloud forming a galaxy) has 4D spin and 4D flow with respect to the independent background/the 4D conformal space-time as shown in Figure 1.

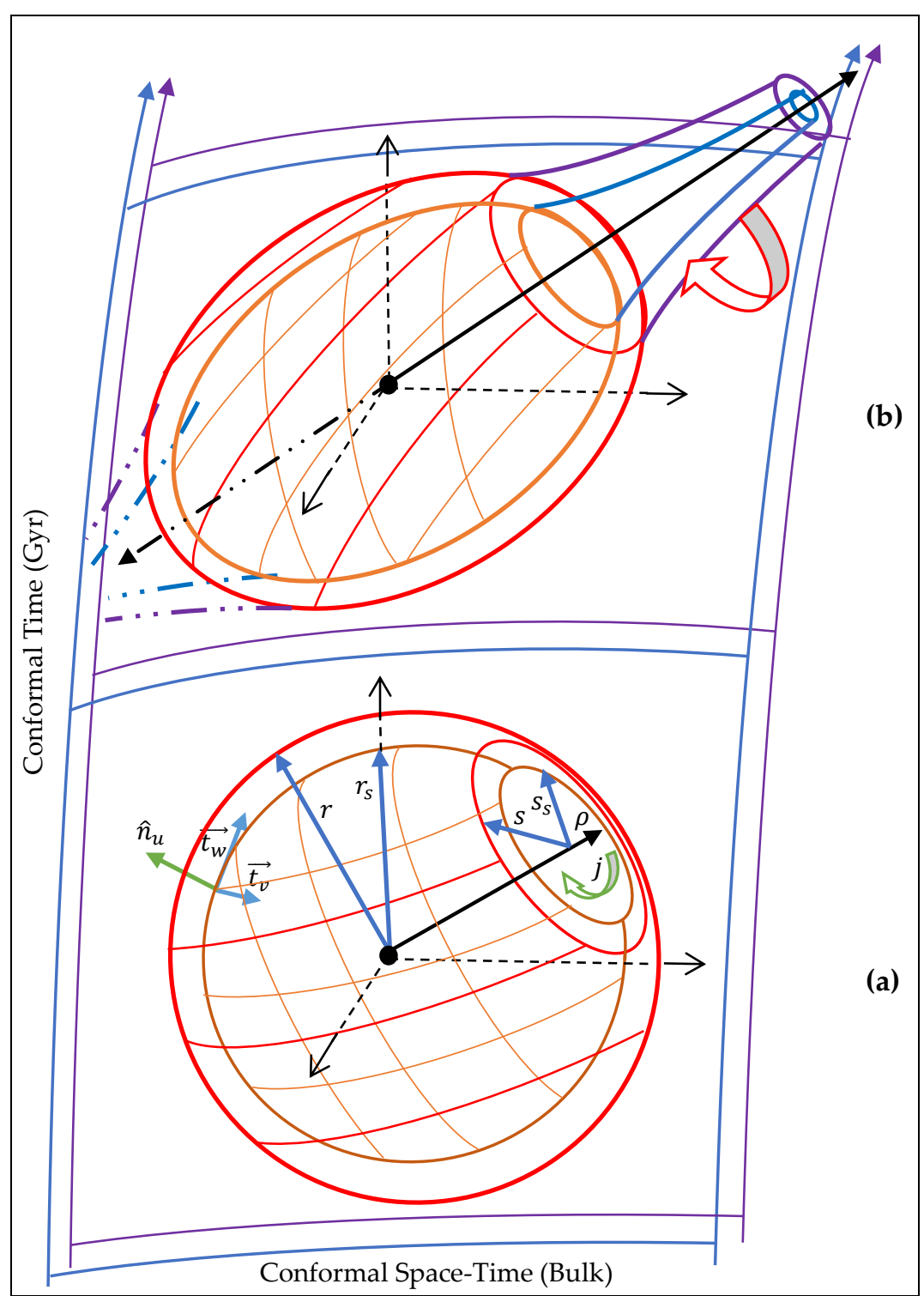

Figure 1. The hypersphere of a compact core of a galaxy (the red-orange $4 \mathrm{D}$ hypersphere/Brane representing the local relativistic space-time) along its travel and spin through the conformal space-time (the blue-purple 4D Bulk representing the independent background of a pre-existing curvature evolving over cosmic time. 
By dividing Equation (10) by the proportionality constant between the stress-energy tensor and the curvature tensor and acquiring the trace reverse equations, we get

$$
R_{\mu \nu}=\frac{1}{2} R \hat{g}_{\mu \nu} \cong 0
$$

where $\hat{g}_{\mu \nu}=g_{\mu \nu}+2 \tilde{q}_{\mu \nu}$ and $\tilde{g}_{\mu \nu}=g_{\mu \nu} \Omega^{2}$. For an ideal case $R_{\mu \nu}=0$, results in Schwarzschild metric as

$$
d s^{2}=\left(1-\frac{r_{s}}{r}\right) c^{2} d t^{2}-\frac{s_{s}{ }^{2}}{s^{2}}\left(\frac{d r^{2}}{1-\frac{r_{s}}{r}}+r^{2} d \theta^{2}+r^{2} \sin ^{2} \theta d \phi^{2}\right)
$$

where $r_{s}$ is the Schwarzschild radius. Since the general form of the conformal time is $d \eta=\Omega^{2} d t$ and by comparing the conformal transformation of the time coordinate in both Equations $(11,12)$ as $\left.g_{t t}+2 \tilde{q}_{t t}=\left(1-r_{s} / r\right)\right) c^{2} d t^{2}$, which shows the conformal function is $\Omega^{2}=-r_{s} / r$, revealing a spatial shrinking through the conformal time agreeing with the vortex model which can occur due to the high spinning, i.e., forming a dual wormhole perpendicular on the galaxy plane that evolve in opposite directions as shown in Figure 1b. To account for this conformal shrinking, a dimensionless spatial scale factor, $s_{s}{ }^{2} / s^{2}$, has been incorporated into the metric in Equation (12). However, for a realistic case when $R_{\mu \nu} \cong 0$, the Kerr metric can be utilized to account for the spinning. A dimensionless spatial scale factor, $s_{s}{ }^{2} / s^{2}$ is incorporating to account for spatial shrinking through conformal time as follows

$$
\begin{aligned}
d s^{2}= & \left(1-\frac{r_{s} r}{\Sigma}\right) c^{2} d t^{2}-\frac{s_{s}{ }^{2}}{s^{2}}\left(\frac{\Sigma}{\Delta} d r^{2}+\Sigma d \theta^{2}+\left(r^{2}+a^{2}+\frac{r_{s} r a^{2}}{\Sigma} \sin ^{2} \theta\right) \sin ^{2} \theta d \phi^{2}\right) \\
& -\frac{2 r_{s} r a \sin ^{2} \theta}{\Sigma} c d t d \phi
\end{aligned}
$$

where $\Sigma=r^{2}+a^{2} \sin ^{2} \theta$ and $\Delta=r^{2}-r_{s} r+a^{2}$ and $a=J / M c ; \quad J$ is the spin momentum.

This scenario of galaxy formation indicates that the core of the galaxy forms a central event horizon leading to opposite traversable wormholes spatially shrinking through the conformal time in the Bulk. The galaxy and its core form at the same process while the gas clouds outside the core would form the spiral arms where the fast-rotating core induces frame dragging [20]. Accordingly, The G2 that faced the drag effects should have its orbit around the wormhole but at a distance from the central event horizon. This scenario that the galaxy and its core form at the same process might explain the formation of supermassive compact cores with a mass of $\sim 10^{9} M_{\odot}$ at just $6 \%$ of the current Universe age [21]; thus, could solve the blackhole hierarchy problem in contrast with a mere supermassive black hole model [22]. Further, the observation of the superluminal motion in the $x$-ray jet of M87 [23] could be travel through these traversable wormholes. These findings are consistent with the observations of high-energy structures perpendicular to the central plane of the disk galaxies [24,25].

To evaluate the influence of the spinning momentum and the curvature of the background on the core of the galaxy and the surrounding gas clouds (the spiral arms), a fluid simulation was performed based on Newtonian dynamics by using the Fluid Pressure and Flow software [26]. In this simulation, the fluid was deemed to represent the space-time continuum throughout incrementally flattening curvature paths representing conformal curvature evolution to analyze the external momenta exerted on objects flowing throughout the incrementally flattening curvatures. The momenta yielded by the fluid simulation were used to simulate a spiral galaxy as a forced vortex. 


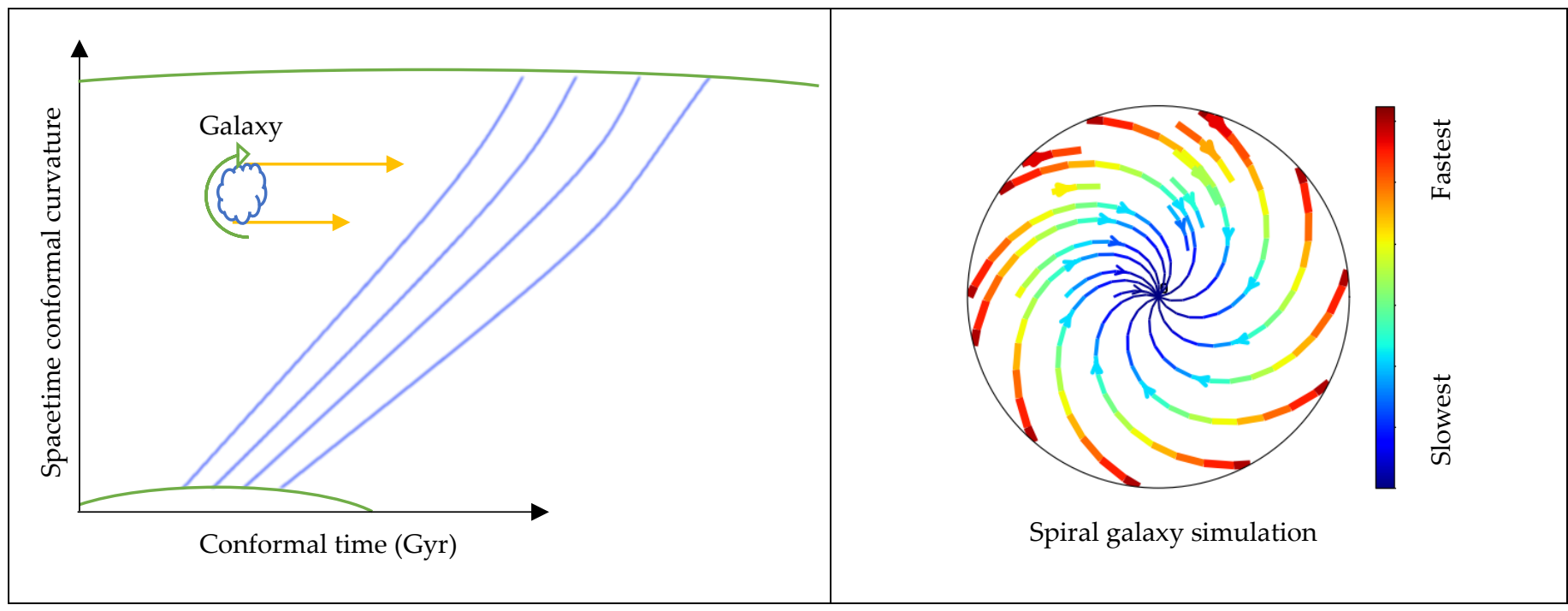

Figure 2. (a) External fields exerted on a galaxy due to the space-time conformal curvature evolution. Green curves represent the curvature of space-time worldlines. Blue curves represent the simulated space-time continuum flux. (b) Simulation of spiral galaxy rotation. Blue represents the slowest tangential speeds and red represents the fastest speeds.

The simulation shows that the tangential speeds of the outer parts of the spiral galaxy are rotating faster in comparison with the rotational speeds of the inner parts, which is consistent with observations of galaxy rotation. Since the rotation speed only depends on the galaxy mass and the background curvature, the findings are consistent with the baryonic Tully-Fisher relation.

\section{Conclusions and Future Works}

In this study, a cloud theory is presented where a galaxy formation scenario is derived in which the Bulk is regarded as independent background as the 4D absolute conformal space-time of distinct curvature that can evolve over cosmic time, where the recent PL18 release has preferred a positively curved early Universe with a confidence level higher than 99\%. Throughout this Bulk, Branes or the 4D relativistic Clouds-Worlds of celestial objects can flow and spin.

The derived field equations were utilized to construct the Brane notion to higher dimensions in the form of a local 4D relativistic spacetime (Brane as the large gas cloud core) of intrinsic curvature and local boundary, which travels and spins with respect to the independent background (Bulk as the 4D conformal space-time) of intrinsic absolute curvature and global extrinsic conformal curvature).

The findings of galaxy formation showed that the core of the galaxy forms as a central vortex with an event horizon leading to opposite traversable wormholes that shrink through the conformal time. It revealed that the galaxy and its core form at the same process, while the gas clouds outside the core would form the spiral arms owning to the fastrotating core that induces the frame-dragging. Orbiting around one of the wormholes and far away from the central event horizon can explain the observation of the G2 cloud which only faced the drag effects. The observation of the superluminal motion in the x-ray jet of M87 could be travel through these traversable wormholes. The formation of the galaxy and its core at the same process could elucidate the formation of supermassive compact galaxy cores with a mass of $\sim 10^{9} \mathrm{M} \odot$ at just $6 \%$ of the current Universe age and might solve the black hole hierarchy problem. The derived field equations should be applied to atoms as well that with galaxies, which can be investigated in future works

Funding: This research received no funding.

Conflicts of Interest: The author declares no conflict of interest. 


\section{References}

[1] Kassin S A, Weiner B J, Faber S M, Gardner J P, Willmer C N A, Coil A L, Cooper M C, Devriendt J, Dutton A A, Guhathakurta P, Koo D C, Metevier A J, Noeske K G and Primack J R 2012 THE EPOCH OF DISK SETTLING: z 1 TO NOW Astrophys. J. 758106

[2] Kassin S A, Brooks A, Governato F, Weiner B J and Gardner J P 2014 KINEMATIC EVOLUTION OF SIMULATED STARFORMING GALAXIES

[3] Di Valentino E, Melchiorri A and Silk J 2020 Planck evidence for a closed Universe and a possible crisis for cosmology Nat. Astron. 4 196-203

[4] Handley W 2021 Curvature tension: Evidence for a closed universe Phys. Rev. D 103 L041301

[5] Chae K H, Lelli F, Desmond H, McGaugh S S, Li P and Schombert J M 2020 Testing the strong equivalence principle: Detection of the external field effect in rotationally supported galaxies arXiv 90451

[6] Kroupa P 2012 The dark matter crisis: Falsification of the current standard model of cosmology Publ. Astron. Soc. Aust. 29 395-433 McGaugh S S 2012 The baryonic tully-fisher relation of gas-rich galaxies as a test of $\Lambda \mathrm{cDM}$ and MOND Astron. J. 14340

[8] Amon A, Gruen D, Troxel M A, MacCrann N, Dodelson S, Choi A, Doux C, Secco L F, Samuroff S, Krause E, Cordero J, Myles J, DeRose J, Wechsler R H, Gatti M, Navarro-Alsina A, Bernstein G M, Jain B, Blazek J, Alarcon A, Ferté A, Raveri M, Lemos P, Campos A, Prat J, Sánchez C, Jarvis M, Alves O, Andrade-Oliveira F, Baxter E, Bechtol K, Becker M R, Bridle S L, Camacho H, Campos A, Rosell A C, Kind M C, Cawthon R, Chang C, Chen R, Chintalapati P, Crocce M, Davis C, Diehl H T, Drlica-Wagner A, Eckert K, Eifler T F, Elvin-Poole J, Everett S, Fang X, Fosalba P, Friedrich O, Giannini G, Gruendl R A, Harrison I, Hartley W G, Herner K, Huang H, Huff E M, Huterer D, Kuropatkin N, Leget P-F, Liddle A R, McCullough J, Muir J, Pandey S, Park Y, Porredon A, Refregier A, Rollins R P, Roodman A, Rosenfeld R, Ross A J, Rykoff E S, Sanchez J, Sevilla-Noarbe I, Sheldon E, Shin T, Troja A, Tutusaus I, Varga T N, Weaverdyck N, Yanny B, Yin B, Zhang Y, Zuntz J, Aguena M, Allam S, Annis J, Bacon D, Bertin E, Bhargava S, Brooks D, Buckley-Geer E, Burke D L, Carretero J, Costanzi M, da Costa L N, et al 2021 Dark Energy Survey Year 3 Results: Cosmology from Cosmic Shear and Robustness to Data Calibration 2168

[9] DES Collaboration, Abbott T M C, Aguena M, Alarcon A, Allam S, Alves O, Amon A, Andrade-Oliveira F, Annis J, Avila S, Bacon D, Baxter E, Bechtol K, Becker M R, Bernstein G M, Bhargava S, Birrer S, Blazek J, Brandao-Souza A, Bridle S L, Brooks D, Buckley-Geer E, Burke D L, Camacho H, Campos A, Rosell A C, Kind M C, Carretero J, Castander F J, Cawthon R, Chang C, Chen A, Chen R, Choi A, Conselice C, Cordero J, Costanzi M, Crocce M, da Costa L N, Pereira M E da S, Davis C, Davis T M, De Vicente J, DeRose J, Desai S, Di Valentino E, Diehl H T, Dietrich J P, Dodelson S, Doel P, Doux C, Drlica-Wagner A, Eckert K, Eifler T F, Elsner F, Elvin-Poole J, Everett S, Evrard A E, Fang X, Farahi A, Fernandez E, Ferrero I, Ferté A, Fosalba P, Friedrich O, Frieman J, García-Bellido J, Gatti M, Gaztanaga E, Gerdes D W, Giannantonio T, Giannini G, Gruen D, Gruendl R A, Gschwend J, Gutierrez G, Harrison I, Hartley W G, Herner K, Hinton S R, Hollowood D L, Honscheid K, Hoyle B, Huff E M, Huterer D, Jain B, James D J, Jarvis M, Jeffrey N, Jeltema T, Kovacs A, Krause E, Kron R, Kuehn K, Kuropatkin N, Lahav O, Leget P-F, Lemos P, et al 2021 Dark Energy Survey Year 3 Results: Cosmological Constraints from Galaxy Clustering and Weak Lensing 5430

[10] Guo Q, Hu H, Zheng Z, Liao S, Du W, Mao S, Jiang L, Wang J, Peng Y, Gao L, Wang J and Wu H 2019 Further evidence for a population of dark-matter-deficient dwarf galaxies Nat. Astron. 4 246-51

[11] van Dokkum P, Danieli S, Abraham R, Conroy C and Romanowsky A J 2019 A Second Galaxy Missing Dark Matter in the NGC 1052 Group Astrophys. J. 874 L5

[12] Danieli S, van Dokkum P, Conroy C, Abraham R and Romanowsky A J 2019 Still Missing Dark Matter: KCWI Highresolution Stellar Kinematics of NGC1052-DF2 Astrophys. J. 874 L12 
[13] Burkert A, Schartmann M, Alig C, Gillessen S, Genzel R, Fritz T K and Eisenhauer F 2012 Physics of the galactic center cloud G2, on its way toward the supermassive black hole Astrophys. J. 75058

[14] Becerra-Vergara E A, Argüelles C R, Krut A, Rueda J A and Ruffini R 2021 Hinting a dark matter nature of Sgr A* via the Sstars Mon. Not. R. Astron. Soc. Lett. 505 L64-8

[15] S. M. Carroll 2003 Spacetime and Geometry: An Introduction to General Relativity

[16] Straub W O 2006 Simple Derivation of the Weyl Conformal Tensor

[17] Kozameh C, Newman E, gravitation K T-G relativity and and 1985 undefined 1985 Conformal Einstein spaces Springer

[18] Straumann N 2013 General Relativity (Graduate Texts in Physics) Springer (Springer)

[19] Pavel Grinfeld 2013 Introduction to Tensor Analysis and the Calculus of Moving Surfaces (Springer)

[20] Krishnan V V, Bailes M, Van Straten W, Wex N, Freire P C C, Keane E F, Tauris T M, Rosado P A, Bhat N D R, Flynn C, Jameson A and Osłowski S 2020 Lense-Thirring frame dragging induced by a fast-rotating white dwarf in a binary pulsar system

[21] Feng W-X, Yu H-B and Zhong Y-M 2021 Seeding Supermassive Black Holes with Self-interacting Dark Matter: A Unified Scenario with Baryons Astrophys. J. Lett. 914 L26

[22] Shen Y 2009 Supermassive black holes in the hierarchical universe: A general framework and observational tests Astrophys. J. $70489-108$

[23] Snios B, Nulsen P E J, Kraft R P, Cheung C C, Meyer E T, Forman W R, Jones C and Murray S S 2019 Detection of Superluminal Motion in the X-Ray Jet of M87

[24] Wang Q D 2021 Chandra large-scale mapping of the Galactic Centre: probing high-energy structures around the central molecular zone Mon. Not. R. Astron. Soc. 504 1609-18

[25] Heywood I, Camilo F, Cotton W D, Yusef-Zadeh F, Abbott D, Adam M, Aldera M A, Bauermeister F, Booth S, Botha A G, Botha D H, Brederode L S, Brits Z B, Buchner S J, Burger J P, Chalmers J M, de Villiers D, Dikgale-Mahlakoana M A, du toit L J, P esterhuyse S W, Fanaroff B L, Foley A, Fourie D J, Gamatham G, Goedhart S, Gounden S, Hlakola M J, Hoek C J, Hokwana A, Horn D M, G Horrell J M, Hugo B, Isaacson A, Jonas J L, B L Jordaan J D, Joubert A F, G Józsa G I, Julie P M, Kapp F B, Kenyon J S, A Kotzé P P, Kriel H, Kusel W, Liebenberg D, Loots A, Lunsky B M, Macfarlane P S, Magnus L G, Magozore C M, Mahgoub O, L Main J P, Malan J A, Malgas D, Manley J, J Maree M D, Merry B, Mnyandu N, Moeng I P, Mphego M C, New W S, Ngcebetsha B, Oozeer N, Otto A J, Passmoor S S, Patel A A, Peens-Hough A, Perkins S J, ratcliffe S M, rust A, Salie S, Schwardt L C, Serylak M, Sirothia S K, Smirnov O M, Sofeya L, Swart P S, tasse C, taylor D, theron I P, thorat K, tiplady A J, tshongweni S, van Balla J, van der Byl A, van der Merwe C, van Dyk C L, rooyen V, Van tonder V, Wyk V, Wallace B H, Welz M G and Williams L P Inflation of 430-parsec bipolar radio bubbles in the Galactic Centre by an energetic event Nature

[26] Reid S, Podolefsky H and Pual A 2013 Fluid Pressure and Flow, PhET Interactive Simulations. 